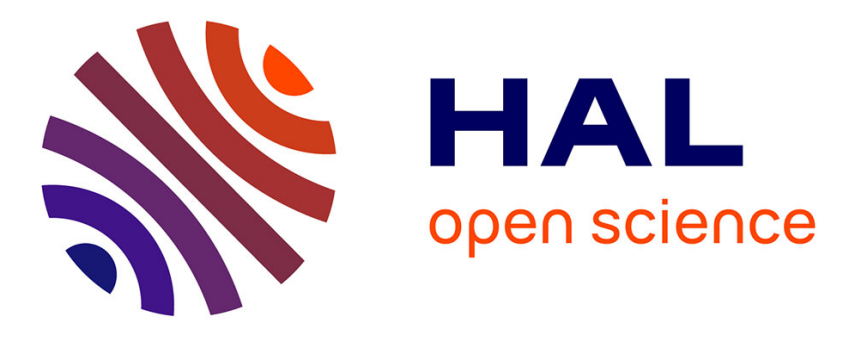

\title{
Surface structure of the Ag-In-(rare earth) complex intermetallics
}

S. S Hars, H.R. Sharma, J. A Smerdon, T. P. Yadav, A. Al-Mahboob, Julian

Ledieu, Vincent Fournée, R. Tamura, R. Mcgrath

\section{To cite this version:}

S. S Hars, H.R. Sharma, J. A Smerdon, T. P. Yadav, A. Al-Mahboob, et al.. Surface structure of the Ag-In-(rare earth) complex intermetallics. Physical Review B: Condensed Matter and Materials Physics (1998-2015), 2016, 93 (20), pp.205428 - 205428. 10.1103/PhysRevB.93.205428 . hal01665251

\section{HAL Id: hal-01665251 \\ https://hal.science/hal-01665251}

Submitted on 15 Dec 2017

HAL is a multi-disciplinary open access archive for the deposit and dissemination of scientific research documents, whether they are published or not. The documents may come from teaching and research institutions in France or abroad, or from public or private research centers.
L'archive ouverte pluridisciplinaire HAL, est destinée au dépôt et à la diffusion de documents scientifiques de niveau recherche, publiés ou non, émanant des établissements d'enseignement et de recherche français ou étrangers, des laboratoires publics ou privés. 


\title{
Surface structure of the Ag-In-(rare earth) complex intermetallics
}

\author{
S. S. Hars, ${ }^{1}$ H. R. Sharma, ${ }^{1, *}$ J. A. Smerdon, ${ }^{1,2}$ T. P. Yadav,${ }^{1,3}$ A. Al-Mahboob, ${ }^{1}$ J. Ledieu, ${ }^{4}$ V. Fournée, ${ }^{4}$ \\ R. Tamura, ${ }^{5}$ and R. McGrath ${ }^{1}$ \\ ${ }^{1}$ Surface Science Research Centre and Department of Physics, The University of Liverpool, Liverpool L69 3BX, United Kingdom \\ ${ }^{2}$ Jeremiah Horrocks Institute for Mathematics, Physics and Astronomy, University of Central Lancashire, Preston, \\ Lancashire PR1 2HE, United Kingdom \\ ${ }^{3}$ Hydrogen Energy Center, Department of Physics, Banaras Hindu University, Varanasi-221005, India \\ ${ }^{4}$ Institut Jean Lamour (UMR7198 CNRS-Université de Lorraine), Parc de Saurupt, 54011 Nancy Cedex, France \\ ${ }^{5}$ Department of Materials Science and Technology, Tokyo University of Science, Niijuku, Tokyo 125-8585, Japan
}

(Received 3 December 2015; revised manuscript received 22 April 2016; published 18 May 2016)

\begin{abstract}
We present a study of the surface structure of the Ag-In-RE (RE: rare-earth elements $\mathrm{Gd}, \mathrm{Tb}$, and $\mathrm{Yb}$ ) complex intermetallics using scanning tunneling microscopy and low-energy electron diffraction. The surface of the Ag-In-Yb approximant prepared by sputter-annealing methods under ultrahigh-vacuum conditions produces a flat (100) surface with no facets. However, the Ag-In-Gd and Ag-In-Tb 1/1 approximants, which have a surface miscut of about $12^{\circ}$ relative to the (100) plane, develop surface facets along various crystallographic directions. The structure of each facet can be explained as a truncation of the rhombic triacontahedral clusters, i.e., the main building blocks of these systems. Despite their differences in atomic structure, symmetry, and density, the facets show common features. The facet planes are In rich. The analysis of the nearest-neighbor atom distances suggests that In atoms form bonds with the RE atoms, which we suggest is a key factor that stabilizes even low-density facet planes.
\end{abstract}

DOI: 10.1103/PhysRevB.93.205428

\section{INTRODUCTION}

Over the past several years, surface science methodologies have developed to the extent that surfaces which are complex both in terms of their structure and composition may be usefully studied. This has enabled materials researchers to investigate the structure and properties of classes of new materials which might previously have proven to be too demanding. The intermetallic compounds (IMCs) are one such class. IMCs contain two or more metallic elements with physical and chemical properties different from the constituent metals and thus offer the possibility of tuning properties for desirable applications. The structure of IMCs can be very complex with up to thousands of atoms per unit cell [1] and include as a subset quasiperiodic structures with infinitely large unit cells [2].

The IMCs discussed here are the so-called approximants to quasicrystals. They possess a complex atomic structure which can be explained in terms of clusters of atoms decorating a periodic lattice. They have large unit cells sometimes containing several hundred atoms. Quasicrystals are formed by the same clusters (building blocks) as approximants, but the clusters are arranged in an aperiodic lattice, possessing aperiodic long-range order and often classically forbidden rotational symmetries. Quasicrystals can be considered as projections of higher-dimension periodic lattices onto threedimensional real space where the tangent of the angle of projection is irrational: $1 / \tau$, where $\tau=1.618 \ldots$ is the golden mean. Approximants are periodic structures projected from the same higher-dimensional lattice but with a rational tangent. The lowest-order approximant is the $1 / 1$, and successively

*H.R.Sharma@liverpool.ac.uk higher-order approximants such as $2 / 1,3 / 2,5 / 3, \ldots$ more closely approach the quasicrystalline structure.

The systems used in this work are Ag-In-(rare earth) (Ag-In-RE) approximants. These systems are promising for various reasons. First, they are available in a suitable form for surface studies and yet in various orders of complexity: 1/1 approximant, 2/1 approximant, and quasicrystal [3-6]. Second, isostructural approximant phases have been observed in a wide range of materials: Ag-In-RE (RE: $\mathrm{Eu}, \mathrm{Gd}, \mathrm{Tb}$, and $\mathrm{Yb}$ ) [3-6] and magnetic Au-Si-Tb [7,8]. This allows comparison of surface phenomena in a wide range of materials with various orders of complexity. Third, these systems are isostructural to the binary $\mathrm{Cd}-\mathrm{Yb}$ approximant systems, where $\mathrm{Cd}$ is replaced by $\mathrm{Ag}$ and $\mathrm{In}$. The bulk structure of the $\mathrm{Cd}-\mathrm{Yb}$ system is well understood [9]. This makes it possible to unambiguously compare the surface structure with a wellestablished model of the bulk. Finally, the structure of these systems is based on rhombic triacontahedral (RTH) clusters $[9,10]$, which are different from the Mackay and Bergman clusters of the Al-based phases. Thus, study of these systems extends the scope of surface studies beyond the commonly used Al-based phases.

We have employed scanning tunneling microscopy (STM) and low-energy electron diffraction (LEED) to characterize the surface structure of three different systems, i.e., Ag-In-RE (RE: Gd, Tb, and $\mathrm{Yb}$ ) 1/1 approximants. We demonstrate that upon standard sputter-annealing preparation, facets along various crystallographic directions develop if the surface has been cut significantly out of alignment with a low-index plane (a so-called miscut). We investigate various factors, such as atomic density, chemistry, bonding between atoms, and symmetry of the clusters, that may influence the stability of the observed facets. Interestingly, we find that the factor which is common in all facets is bonding between In and RE atoms. 
We suggest that this bonding is a key factor that stabilizes even low atomic density facets.

While small miscuts are often employed to alter surface properties, such as increasing the density of step edges, the large miscut employed here provides the opportunity to study the relative stability of the crystallographic facet surfaces. The experiment offers an insight into what could happen for intermetallic compound nanoparticles where facets are likely to develop. Hence it is important to understand the atomic structure for each of the most frequently found facets and, more generally, establish the trend/rules expected for this material. The understanding of surface and interface phenomena is helpful for a knowledge-based optimization of surface properties of IMCs for their uses in technological applications such as heterogeneous catalysts, coatings, and thin films. IMCs are potentially relevant as catalysts because of their tunable electronic properties, isolation of active sites, and low cost [11].

The work also shows that it is possible to generate stable surfaces of varying structural complexity. This could stimulate further studies where the surface properties would be analyzed for a given ternary system as a function of the size of the surface unit cell. For example, different networks of adsorption sites present on the surfaces of IMCs offer the opportunity to explore unprecedented epitaxial phenomena such as threedimensional quasicrystalline films of single elements [12] and quasicrystalline monolayers of molecules $[13,14]$, which can occur when the size of the adsorbing species is compatible with the characteristic length of a specific network of adsorption sites. The current work opens up the opportunity to compare these surface phenomena on related (periodic) approximant surfaces.

\section{EXPERIMENTAL PROCEDURE}

The samples were grown with the self-flux method, starting with $99.99 \%$ pure $\mathrm{Ag}, 99.99 \%$ pure In, and $99.9 \%$ pure $\mathrm{Gd}$, $\mathrm{Tb}$, and $\mathrm{Yb}$. The crystal composition of the samples was $\mathrm{Ag}_{46.5} \operatorname{In}_{39.2} \mathrm{Gd}_{14.3}, \mathrm{Ag}_{47} \mathrm{In}_{38.3} \mathrm{~Tb}_{14.7}$, and $\mathrm{Ag}_{39.3} \mathrm{In}_{45.9} \mathrm{Yb}_{14.8}$. The detail of the growth method is explained elsewhere $[6,15]$.

The samples naturally grow with a (100) surface orientation. For these surface studies, the Ag-In-Gd and AgIn-Tb samples were hand polished along (100) facet using diamond paste of 6,1 , and $0.25 \mu \mathrm{m}$ grades successively. The crystallographic orientation of the Ag-In-Yb sample was determined by Laue backscattering and then cut along the (100) surface by spark erosion. This method was not possible for the other systems because of the small size of the samples. The surfaces were then cleaned in ultrahigh vacuum (UHV) by sputtering at room temperature with $\mathrm{Ar}^{+}$ions at $2.5-3 \mathrm{keV}$ for 30 minutes, followed by annealing at $450{ }^{\circ} \mathrm{C}$ for four hours each cycle. An infrared pyrometer with emissivity set to 0.35 was used to monitor the temperature.

Both STM images and LEED patterns were recorded at room temperature using an Omicron instrument. STM images were analyzed using WSXM software [16]. STM images presented here were subject to a minimal degree of skew to correct the thermal drift. VESTA was used to analyze the structural model [17].

\section{RESULTS}

\section{A. Bulk structure of the Ag-In-RE approximants}

Before we present the experimental results, we describe the bulk structure of the Ag-In-RE 1/1 approximant. The Ag-In-RE 1/1 approximant is isostructural to the Cd-RE $1 / 1$ approximant, where $\mathrm{Cd}$ is replaced by $\mathrm{Ag}$ and $\mathrm{In}$. The structure can be described as a body-centered-cubic packing of rhombic triacontahedral (RTH) clusters. The RTH cluster consists of five successive shells (Fig. 1). The innermost shell has $\mathrm{Cd}$ atoms forming a tetrahedron. Twenty $\mathrm{Cd}$ atoms in the second shell make a dodecahedron. The third shell is an icosahedron consisting of $12 \mathrm{RE}$ atoms. The fourth shell is an icosidodecahedron consisting of $30 \mathrm{Cd}$ atoms. Finally, the outermost shell is a rhombic triacontahedron with $92 \mathrm{Cd}$ atoms located on the vertices and midedges [9]. The diameter of the RTH cluster (measured along the twofold axis) is the same as the lattice constant so that the outermost shells of neighboring clusters share atoms with each other.

The RTH cluster has fivefold, threefold, and twofold axes as seen in Fig. 1(b). For clarity, we have shown only the icosahedral shell of the RTH cluster in the figure. The twofold axis of the cluster is aligned along the [100] direction of the crystal lattice and the threefold axis of the cluster is along [310].

The lattice constant $(a)$ of the three approximants (Cd-RE) is slightly different (Cd-Gd: $15.52 \AA$ [6], Cd-Tb: $15.57 \AA$ [6], and Cd-Yb: $15.66 \AA$ [9]). The size of the shells of the RTH cluster in the three approximants will also be different in the same proportion. For simplicity, we have used the parameters of the $\mathrm{Cd}-\mathrm{Tb} 1 / 1$ approximant throughout the manuscript. The difference in the lattice constant in the three systems is within the uncertainty of the STM measurements.

As mentioned above, $\mathrm{Cd}$ atoms are replaced by $\mathrm{Ag}$ and In in the Ag-In-RE approximant. However, the chemical ordering of $\mathrm{Ag}$ and In has not yet been determined. It is known for $\mathrm{Ag}-\mathrm{In}-\mathrm{Yb}$ that the first shell of the RTH cluster is formed by In atoms. Similarly, the second shell contains $60 \% \mathrm{Ag}$ and $40 \% \mathrm{In}$, and the fourth shell contains $20 \% \mathrm{Ag}$ and $80 \% \mathrm{In}$. The midedge of the fifth shell is $100 \% \mathrm{Ag}$ and vertices contain $37 \%$ $\mathrm{Ag}$ and $63 \%$ In [18]. Similar information for $\mathrm{Ag}-\mathrm{In}-\mathrm{Gd} / \mathrm{Tb}$ is unavailable. Therefore, we will use this information on Ag-In$\mathrm{Yb}$ to infer the composition of the surface layers in Sec. IV. The amount of In in the Ag-In-Gd/Tb bulk is slightly less than in Ag-In-Yb. Therefore, the amount of In estimated for the Ag-In-Gd/Tb surface may be slightly higher than the actual value. However, this will not influence the overall conclusion.

\section{B. Observation and identification of facets}

The surfaces of both Ag-In-Gd and Ag-In-Tb samples after sputtering and annealing display facets along different crystallographic directions (Fig. 2). To find the Miller indices $(h k l)$ of the facet planes, we first identified the (100) facet from its symmetry. Then, the angle between the (100) facet and the other facets was determined using STM images. The calculated angles are compared with the expected values (Table I). The angle alone is not sufficient to specify the Miller indices of the facets. For example, the (310), (310), (301), and (301) planes make the same angle of $18.4^{\circ}$ with the (100) plane. The Miller 

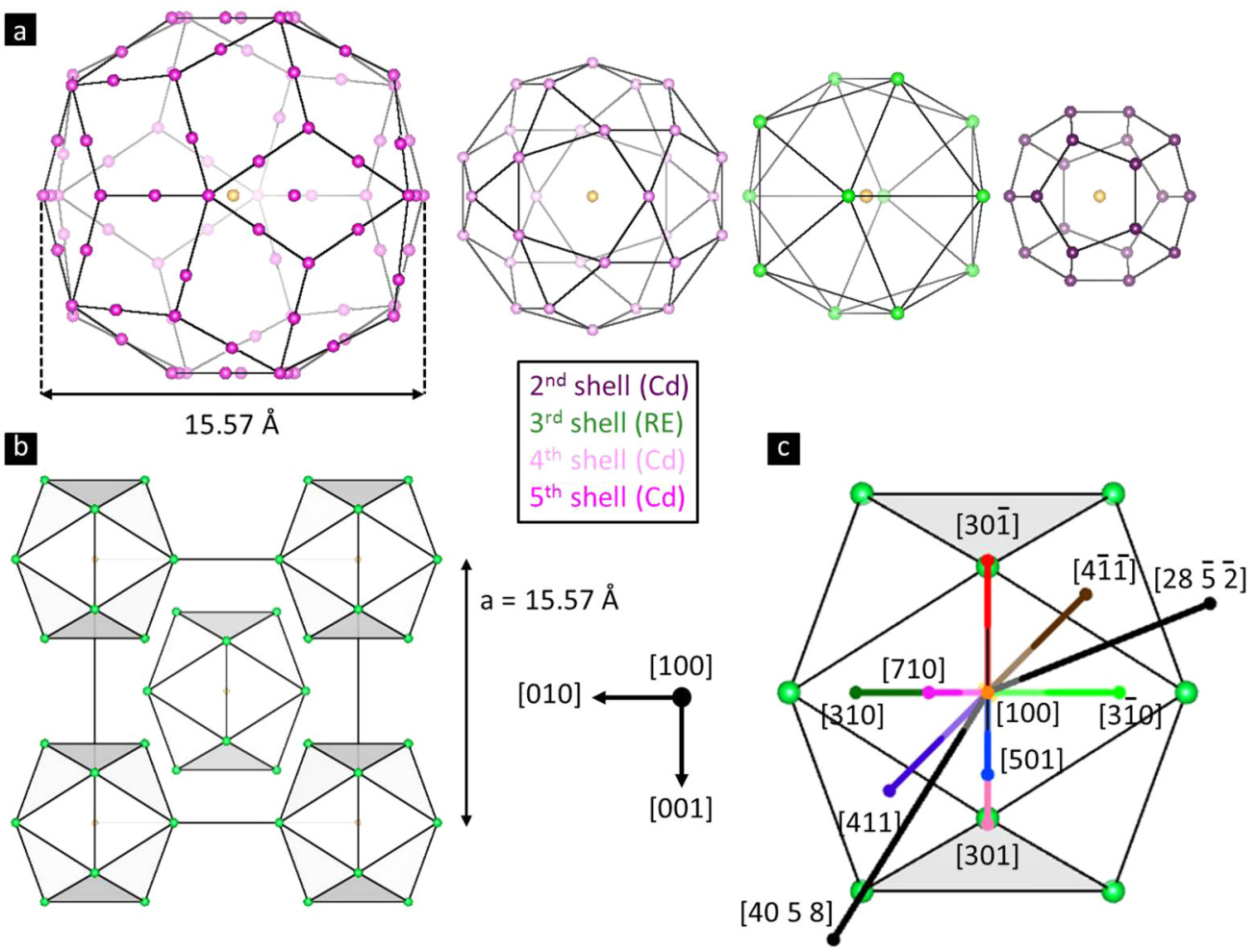

FIG. 1. Structure of the Cd-RE 1/1 approximant. (a) Successive shells of the rhombic triacontahedral (RTH) cluster, which is the building block of the Cd-RE 1/1 approximant (innermost shell is not shown) $[9,10]$. (b) Clusters packed in a bcc lattice. For clarity, only the icosahedral shell of the cluster is shown. (c) Magnified view of the icosahedral shell. The direction of the facets observed in STM are labeled and and indicated in different colors. The center of the RTH cluster is marked by a gold dot. The given lattice parameters are from the Cd-Tb $1 / 1$ approximant.

indices were therefore determined by the cross product of two vectors in the facet plane.

In Ag-In-Gd, the observed facets are the (100), (310), (31̄0), (301), and (4 $\overline{1} \overline{1})$ planes. The Ag-In-Tb sample shows (100), (310), (301), (411), (501), and (710) facets (refer to Table I). The (100) and (310) facets are more frequently observed than the others. For both samples, the (310) facet is equivalent to (310) in terms of atomic structure, but it is different from (301)

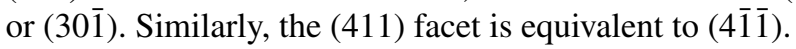

The formation of the facets is further confirmed by comparing STM images with the bulk structure and with LEED patterns. The LEED results will be discussed in Sec. III D. Figure 3(a) shows the lattice planes of the bcc crystal along the observed facets. Here we consider only the space lattice, not the RTH cluster. In Fig. 3(b), we show fast Fourier transform (FFT) of STM images from each facet for comparison. The real-space lattice parameters are calculated from the FFT using the relation $\left|\mathbf{a}_{i}\right|=2 \pi /\left|\mathbf{a}_{i}^{*}\right| \sin \theta$, where $i=1,2, \mathbf{a}_{1}^{*}$ and $\mathbf{a}_{2}^{*}$ are the unit vectors in the FFT, and $\theta$ is the angle between the unit vectors [these vectors are given in Fig. 3(b)]. The FFTs confirm that the symmetry and dimension of the unit cells observed by STM are in agreement with that of the facet planes expected from the bcc structure with lattice constant $a=15.57 \AA$. Atomic resolution in STM was achieved only on the (301) and (501) facets. The other facets exhibit bigger features of larger than $10 \AA$ size. The detailed structure of each facet will be discussed in Sec. III C.

We also analyze whether the facets are aligned along particular high-symmetry directions of the RTH cluster. The surface-normal direction of each observed facet is shown in Fig. 1(c). As can be seen, the (100) and (310) or (310) facets, which were observed more frequently than others, are aligned
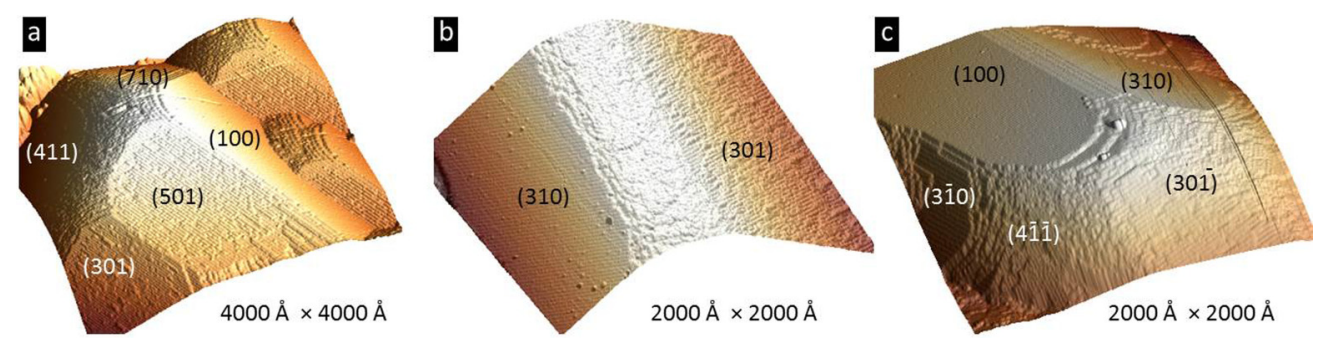

FIG. 2. STM images of facets. STM images from the surface of the Ag-In-Tb (a) $4000 \times 4000 \AA$ and (b) $2000 \times 2000 \AA$, and Ag-In-Gd (c) $2000 \times 2000 \AA 1 / 1$ approximants displaying facets along different crystallographic directions. 
TABLE I. List of facets. The observed facets and their angle of inclination with the (100) surface.

\begin{tabular}{lccccccccc}
\hline \hline Observed facets & $(100)$ & $(310)$ & $(3 \overline{1} 0)$ & $(301)$ & $(30 \overline{1})$ & $(411)$ & $(4 \overline{1} \overline{1})$ & $(501)$ & $(710)$ \\
\hline Expected angle & $0^{\circ}$ & $18.4^{\circ}$ & $18.4^{\circ}$ & $18.4^{\circ}$ & $18.4^{\circ}$ & $19.5^{\circ}$ & $19.5^{\circ}$ & $11.3^{\circ}$ & $8.3^{\circ}$ \\
Measured angle & $0^{\circ}$ & $19.0 \pm 0.5^{\circ}$ & $18.0 \pm 0.3^{\circ}$ & $18.3 \pm 0.2^{\circ}$ & $18.7 \pm 0.1^{\circ}$ & $20.4 \pm 0.2^{\circ}$ & $20.1 \pm 0.2^{\circ}$ & $12.1 \pm 0.2^{\circ}$ & $9.2 \pm 0.4^{\circ}$ \\
Ag-In-Tb & Yes & Yes & No & Yes & No & Yes & No & Yes & Yes \\
Ag-In-Gd & Yes & Yes & Yes & No & Yes & No & Yes & No & No \\
\hline \hline
\end{tabular}

perpendicular to the twofold and threefold axes, respectively. However, the other facet planes are not aligned with any particular high-symmetry direction of the cluster.

The observation of the facets is in contrast to the result from the (100) surface of the isostructural Ag-In-Yb 1/1 approximant. The latter system showed only the flat surface with no facets (refer to Sec. III D). In order to understand the formation of the facets in the other systems, we checked the macroscopic orientation of the surface by Laue backscattering after the UHV measurements were performed. The Laue measurements confirmed that the samples were single grain and that the surfaces were subject to a miscut. The Ag-In-Gd sample has a miscut resulting in a surface at $\left(10^{\circ}, 4^{\circ}\right)$ from the (100) plane (a magnitude of $10.8^{\circ}$ ) and the Ag-In-Tb has a miscut resulting in a surface at $\left(11^{\circ}, 7^{\circ}\right)$ from the (100) plane (a magnitude of $13.1^{\circ}$ ). From these angles, we determine the surface orientation to be close to the $\left\{\begin{array}{lll}28 & 5 & 2\end{array}\right\}$ - and $\{4058\}$-equivalent directions for the Ag-In-Gd and Ag-In-Tb samples, respectively. Among many equivalent directions, (28 5 2) and (40 5 8) would explain why the facets are observed predominantly in a particular direction of the crystal. As can be seen in Fig. 1(c) or Table I, the facets in Ag-In-Gd are observed more towards the $[0 \overline{1} \overline{1}]$ direction than [011], and vice versa in Ag-In-Tb. The miscut is introduced in the first stage of preparing the sample surfaces and, from the evidence at hand, promotes the formation of facets with average orientation matching the miscut.

We have also examined whether a different surfacepreparation method would influence the surface morphology. For this preparation, samples were taken out of the UHV chamber and repolished. The polishing was gently performed in order to avoid introduction of a further miscut. The surface was then sputtered at a high temperature of $450{ }^{\circ} \mathrm{C}$ for several hours with $\mathrm{Ar}^{+}$ions at $2.5-3 \mathrm{keV}$ beam energy. It was then cooled down to room temperature, sputtered briefly (15 minutes with the same parameters) and annealed at $450{ }^{\circ} \mathrm{C}$ for 60 minutes. In the usual preparation method, as described in Sec. II, the surface was sputtered at room temperature for 30 minutes, subsequently annealed at $450{ }^{\circ} \mathrm{C}$ for four hours, and cooled down to room temperature before measurement. The overall facet morphology remained unchanged after this preparation.

\section{Detailed structure of the facets 1. The (100) facet}

STM images from the (100) facet of both Ag-In-Tb and Ag-In-Gd approximants are very similar. Both systems show steps of $\sim 7.50 \AA$ [Fig. 4(a)]. In this section, we show only images from Ag-In-Tb (100). STM images on the terraces show protrusions arranged in a square lattice of $15.01 \pm 0.04 \AA$ size [Fig. 4(b)]. The distance between protrusions is obtained by measuring the peak-to-peak distance between adjacent maxima in the line profiles. The diameter of the protrusions is about $10 \AA$, which is much larger than the usual size of a feature resulting from a single atom. This means that atomic resolution is not obtained in STM. Some of the protrusions are missing from the lattice, indicating vacancy defects [marked by squares in Fig. 4(a)]. The terraces in the (100) surface of the a

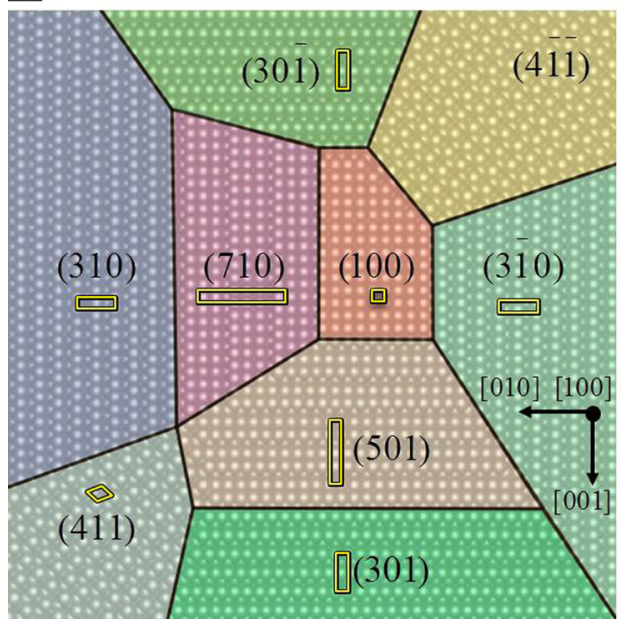

b
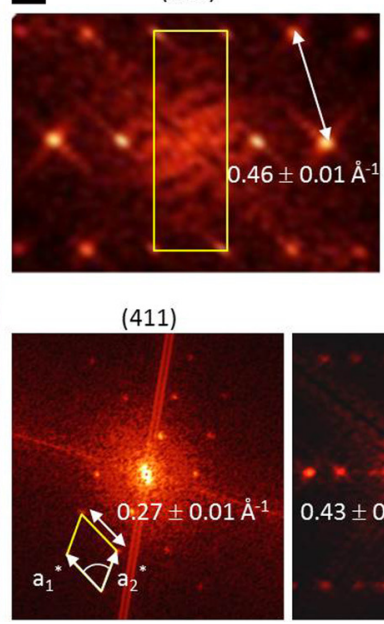

$(100)$

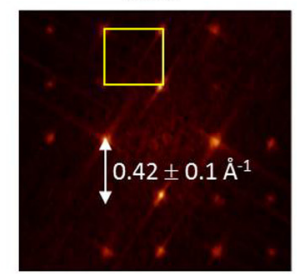

(710)

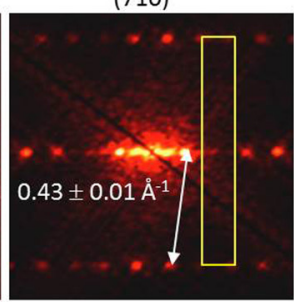

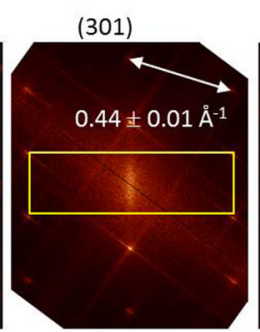

(310)

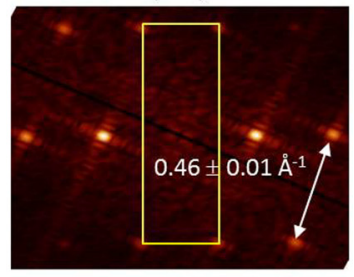

(501)

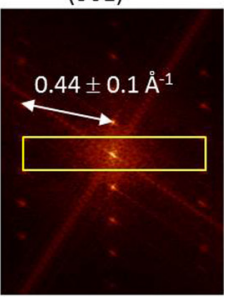

FIG. 3. Symmetry of the facets. (a) A bcc lattice showing truncated planes along the direction of the facets observed by STM in a bird's-eye view along [100]. (b) Fast Fourier transforms (FFTs) of STM images from the facets. The surface unit cells of the facets are marked. The size of the FFTs is not to scale. The intensity of spots is slightly enhanced by Fourier filtering. 

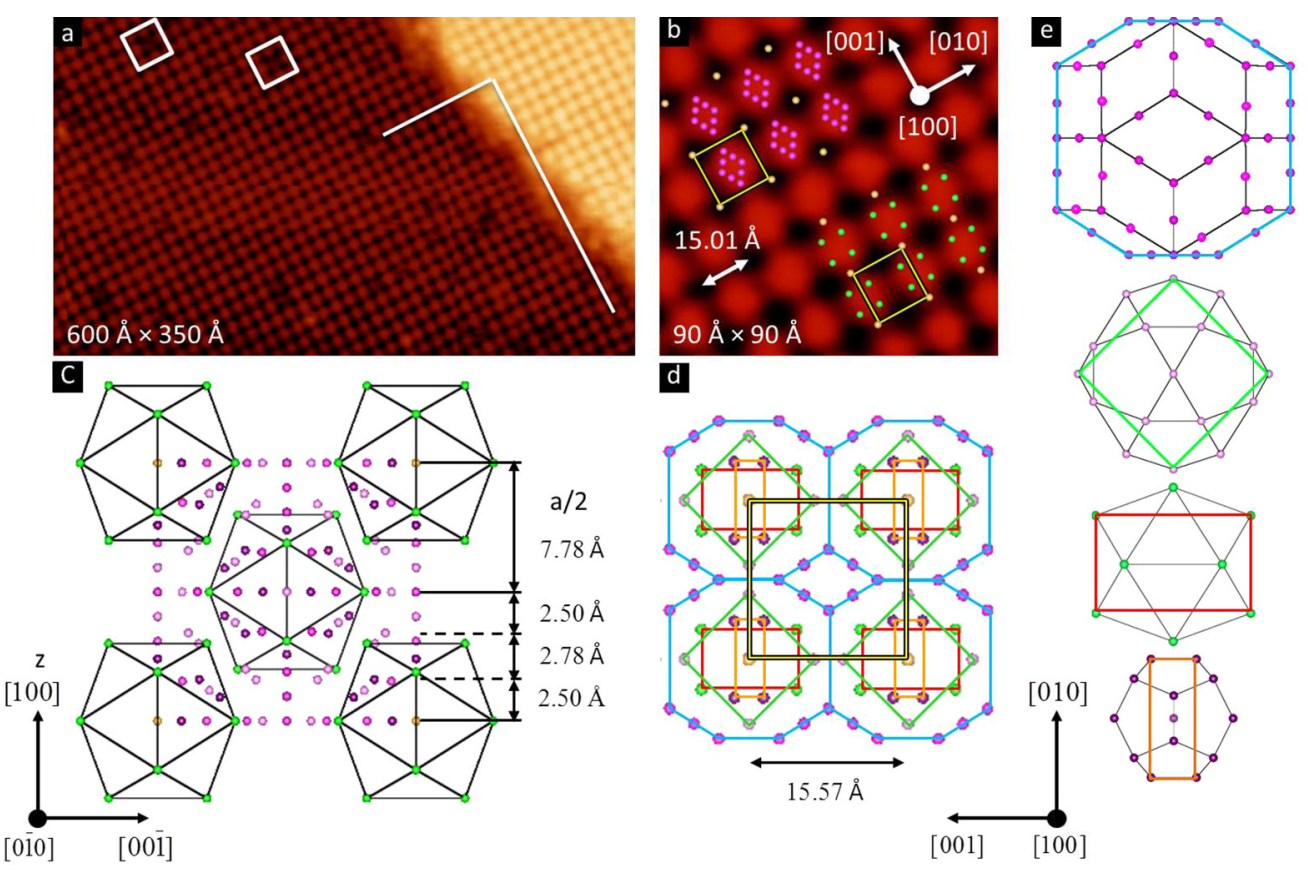

FIG. 4. (100) facet. (a) STM image from the (100) facet of the Ag-In-Tb 1/1 approximant demonstrating two terraces separated by a step of height equal to half the lattice constant $(600 \times 350 \AA)$. Squares mark defects. White lines indicate that rows in the adjacent terraces are shifted by half the row spacing. (b) Magnified view of a terrace $(90 \times 90 \AA$ A). (c) The bulk unit cell of the Cd-RE $1 / 1$ approximant projected normal to [100]. (d) Atomic structure of the (100) plane intersecting the center of the RTH clusters. Atoms belonging to different shells of the RTH clusters are marked. Atoms of the innermost shell, the tetragon, are not considered. The atomic structure of the plane is overlaid on the STM image in (b); only selected shells are shown. (e) Four successive shells of the RTH cluster. The innermost shell is not shown. The color scheme for atoms is the same as in Fig. 1.

Ag-In-Yb 1/1 approximant also exhibit protrusions of similar size and distribution.

In order to explain the step height, we present the bulk unit cell of the Cd-Tb 1/1 approximant in Fig. 4(c). Two different types of layers, i.e., flat and puckered, can be identified perpendicular to the [100] direction. The flat layers are located at $Z=0$ and $Z=a / 2$, where they intersect the center of the RTH cluster. The puckered layers are at $Z=2.50 \AA$ and $Z=5.28 \AA$. Other layers located at $Z>a / 2$ are the mirror reflections of the ones located at $Z<a / 2$.

A previous study on the (100) surface of the Ag-In-Yb 1/1 approximant showed steps of three different heights, 2.1, 3.1, and $7.3 \AA$, which were explained by the formation of terraces at both puckered and flat layers [19]. However, the puckered layers were found to be less stable such that these layers did not appear after the surface was annealed at higher temperatures. The current systems exhibit only one step height of about $7.5 \AA$ under the applied surface-preparation conditions. Based on the observation of the single steps as well as the fact that the terrace structure is similar to that of the Ag-In-Yb approximant, we assume that the current systems also yield terraces at the flat layers, which are denser than the puckered layers.

Because of the lack of atomic resolution, it is not possible to unambiguously relate the protrusions in STM to the atomic structure of the model. However, there are two possible ways to explain the protrusions. In order to illustrate this, we present the structure of the (100) plane intersecting the center of the RTH clusters in Fig. 4(d). Atoms belonging to different shells of the RTH cluster are marked. As explained above, the [100] direction is along the twofold axis of the RTH cluster, and the surface truncated shells show twofold symmetry around the vertices of the unit cell.

The atomic structure is superimposed on the STM images in the two possible options in Fig. 4(b). The first case is that which would be observed if RE atoms of the third shell contribute to the tunneling current producing protrusions. The other case is that the atoms of the outermost shell of the four neighboring RTH clusters, which appear as a diamond in the center of the unit cell, would contribute to the tunneling current producing the protrusions. A report on STM simulation from the (100) surface of a similar system, i.e., the Ag-In-Ca 1/1 approximant [20], shows that In atoms produce a brighter contrast than $\mathrm{Ag}$ and $\mathrm{Ca}$ atoms in STM. If the same is applicable in the current systems, the protrusions are likely to be related to atoms of the outermost shell, as atoms at the vertices of the diamond are mostly In.

We also observe that the rows of protrusions in the adjacent terraces are shifted by half of the lattice constant in the direction normal to the rows, as indicated by white lines in Fig. 4(a). This is expected in a bcc structure if the terraces are formed at the lattice planes intersecting the body center and faces of the cube.

\section{The (310) and (31̄0) facets}

As for the (100) facets, the (310) facets exhibit flat terraces separated by steps [Fig. 5(a)]. The step height is $5.0 \pm 0.3 \AA$, which corresponds to the periodicity expected along [310], which is $a / \sqrt{10}\left(=a / \sqrt{h^{2}+k^{2}+l^{2}}\right)$ [Fig. 5(c)]. The terraces reveal two different types of parallel rows [Fig. 5(b)]. One 

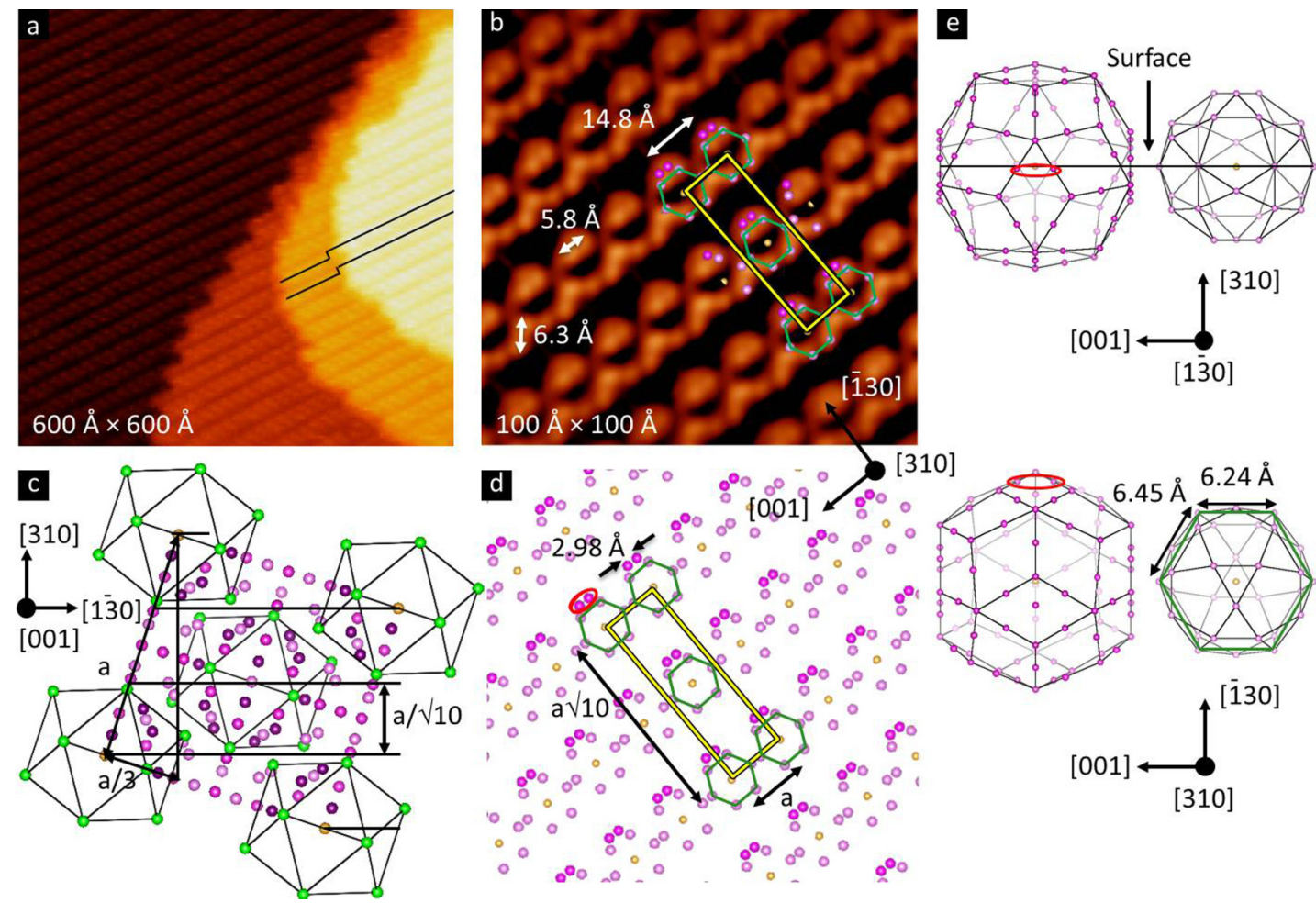

FIG. 5. (310) facet. (a) STM image from the (310) facet of the Ag-In-Gd $1 / 1$ approximant $(600 \times 600 \AA)$. Lines indicate a shift of rows in the adjacent terraces. (b) High-resolution STM image from a terrace of the Ag-In-Tb 1/1 approximant $(100 \times 100 \AA)$. The bulk unit cell of the Cd-RE 1/1 approximant projected normal to [310]. (d) Atomic structure of the (310) plane intersecting the center of the RTH clusters. The Ag/In atoms marked by a hexagon belong to the fourth shell of the RTH cluster. The atomic structure of the plane is overlaid on the STM image in (b). (e) Fifth and fourth shells of the RTH cluster in side and top view. Atoms appearing in the surface layer are marked by a hexagon. The same atoms are marked by red circles. The color scheme for atoms is the same as in Fig. 1.

row exhibits fine features forming a zigzag pattern and the other row consists of protrusions. Both rows show atomic scale resolution. The fine features in the zigzag row are separated by a distance of $6.3 \pm 0.2 \AA$. Two brighter spots are observed within the protrusion, which are at a distance of $5.8 \pm 0.3 \AA$.

The structure in the terraces is consistent with the atomic structure of the plane intersecting the center of the RTH cluster, which is shown in Fig. 5(d). The surface unit cell is a centered rectangle, with lattice points considered at the center of the RTH cluster. The lattice points are decorated by Ag/In atoms forming a hexagon. The hexagons belong to the fourth shell of the cluster [Fig. 5(d)]. In order to interpret the STM images, we also need to consider atoms from the layer underneath, which is $0.2 \AA$ below the top layer. These atoms belong to the fifth shell and line up along one side of the hexagons, i.e., along the [001] direction. The atomic structure is superimposed on the STM image in Fig. 5(b). As can be seen, almost all of the features of the two rows can be explained by the atomic structure. The zigzag pattern is formed by atoms of the fourth shell, which will be mostly In, whereas the protrusions are contributed by the fourth and fifth shell atoms.

For the bcc lattice, the rows in the adjacent terraces are expected to be shifted perpendicular to the rows, i.e., along [130], by $2 a / \sqrt{10}$. The ratio between the shift and the separation of the rows, which is $(a \sqrt{10}) / 2$, is $2 / 5$. This shift is observed in STM, as indicated by lines in Fig. 4(a), further confirming the formation of the (310) facets.
The (310) and (310) facets are equivalent in atomic structure. As the (310) facets are rarely observed and the area of the facets is very small, we were unable to obtain atomic resolution in STM from these facets. However, we could confirm from the analysis of FFT that this facet showed the same unit cell as the (310) facet [Fig. 3(b)].

\section{The (501) facet}

STM images from the (501) facets also reveal a step-terrace structure. The step height is found to be $3.0 \pm 0.1 \AA$, which corresponds to the periodicity expected along [501], i.e., $a / \sqrt{5^{2}+0^{2}+1^{2}}=a / \sqrt{26}$. A high-resolution STM image of a terrace is shown in Fig. 6(a). It exhibits rows of bright dots (indicated by black arrows). The rows are separated by $40.0 \pm 0.4 \AA$. Faint dots are also observed in rows between the bright rows (indicated by white arrows). The dots in both bright and faint rows are paired. The separation of the bright dots in each pair is $5.8 \pm 0.2 \AA$. The bright dots are higher than the faint dots by $0.5 \pm 0.1 \AA$. In addition to the rows, a band of fine structure is apparent between the bright and faint rows. A few other features, for example, those indicated by a dotted arrow, do not appear regularly. The dots can be assigned to atoms. However, the structure in the band is not clear because of poor resolution.

Most of the STM features on the terraces can be explained by the atomic structure of a plane which intersects at about 

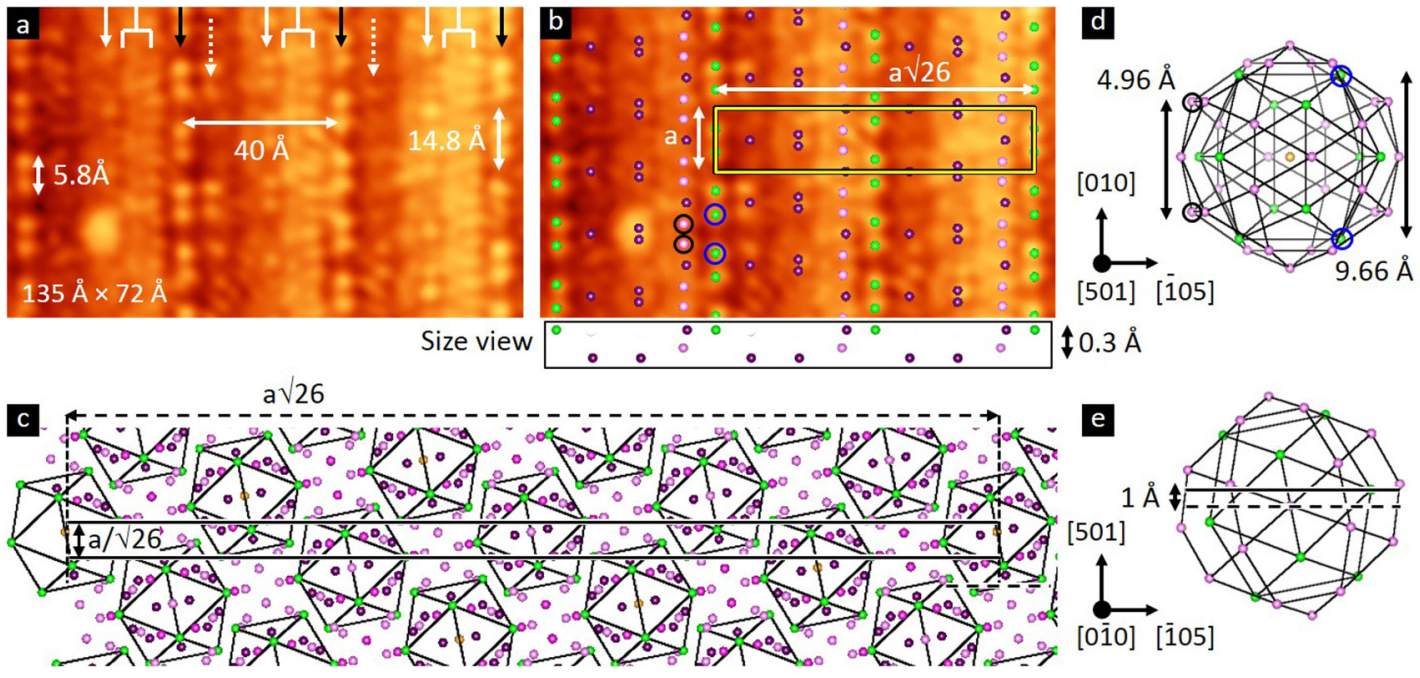

FIG. 6. (501) facet. (a) STM image from the (501) facet in the Ag-In-Tb $1 / 1$ approximant $(135 \times 72 \AA)$. (b) STM image (a) superimposed with the atomic structure of the plane intersecting at $1 \AA$ from the cluster center. (c) The bulk unit cell of the Cd-Tb 1/1 approximant projected normal to [301]. The surface terminating planes are marked by horizontal solid lines. (e) Fourth and third shells of the RTH cluster in side and top view. Atoms appearing in the surface layer are marked. The color scheme for atoms is the same as in Fig. 1.

$1 \AA$ above the cluster center. This plane is marked by a line in Figs. 6(c) and 6(e). The atomic structure of the plane is superimposed on STM in Fig. 6(b). Here, we consider atoms from layers underneath as well. The lowermost layer is at $0.3 \AA$ from the top layer [see side view in Fig. 6(b), bottom]. The surface unit cell is a centered rectangle with dimensions $(a, \sqrt{26} a)$. The position of RE atoms matches exactly that of the bright dots in STM. The faint dots coincide with $\mathrm{Ag} / \mathrm{In}$ atoms from the second shell. The dots in the pairs are slightly displaced from the center of the pair along [010], compared to the model. Similarly, atoms (mostly In) from the fourth and second shells lie along the edge of the band of fine features. The remaining atoms from the second shell, which lie in the lower layer, are either not detected by STM or displaced along [010].

The observed difference in height between the bright and faint dots is consistent with the fact that the bright dots are from the top-layer atoms, while the faint dots are from the lower-layer atoms. Since the other planes in the model do not match with the STM images shown in Figs. 6(a) and 6(b), the specific plane at about $1 \AA$ above the cluster center best matches with the data.

\section{The (301) and (301) facets}

As expected from the bcc lattice structure, STM from the (301) or (301) facets reveals the same symmetry and periodicity as the (310) facets, but the detailed atomic structure is different. This facet also shows parallel rows [Fig. 7(a)]. Each row consists of protrusions (indicated by a black arrow) and dots (indicated by a white arrow). Both protrusions and dots can be assigned to the atomic structure of the plane which intersects $0.28 \AA$ above the cluster center. The atomic structure is superimposed on the STM image in Fig. 7(b). The protrusions match with atoms of the fourth shell, which are mostly In again. The dots coincide with the second shell Ag/In
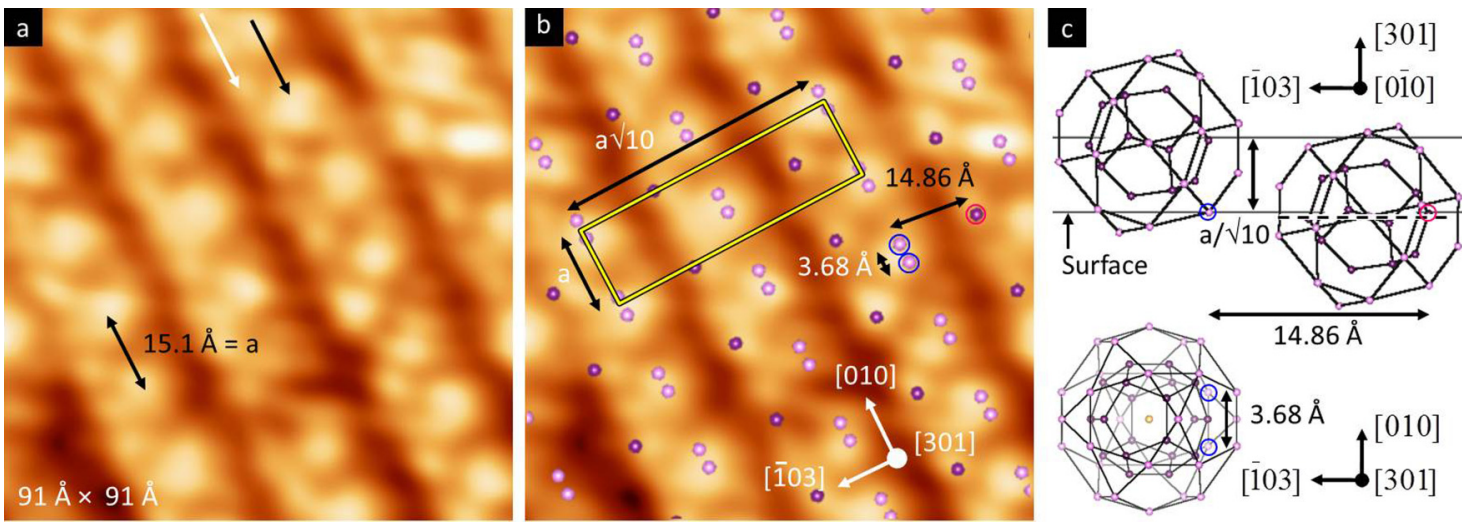

FIG. 7. (301) facet. (a) STM image from the (301) facet in the Ag-In-Tb $1 / 1$ approximant $(91 \times 91 \AA$ ). (b) Image (a) superimposed with the atomic structure of the plane intersecting at $0.28 \AA$ from the cluster center. (c) Fourth and second shells of the RTH cluster in side and top view. Atoms appearing in the surface are marked. The surface terminating planes are indicated by horizontal solid lines. The color scheme for atoms is the same as in Fig. 1. 

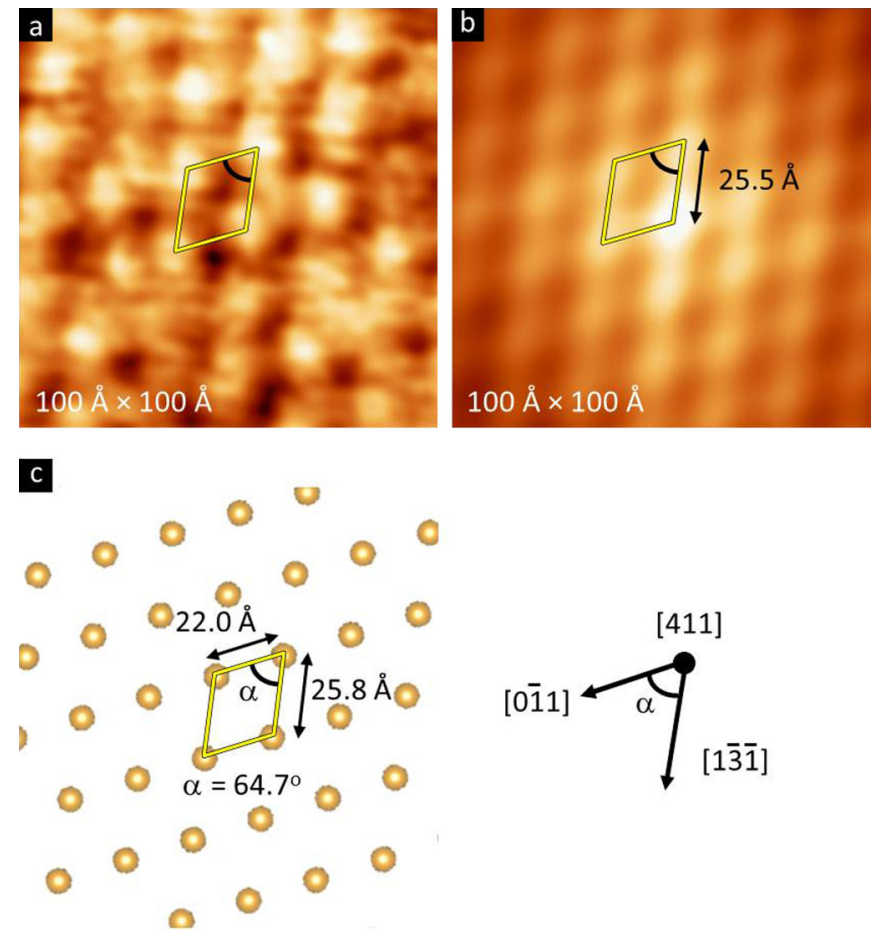

FIG. 8. (411) facet. (a) STM image from the (411) facet in the Ag-In-Tb approximant $(120 \times 120 \AA)$. (b) Autocorrelation function of the STM image in (a). (c) The space lattice of the bcc structure in the (411) plane.

atoms. The unit cell is a centered rectangle of dimension ( $a$, $\sqrt{10} a$ ) as for the (310) facet. The position of the dots matches with that of the second shell atoms, while atoms from the fourth shell coincide with the protrusions.

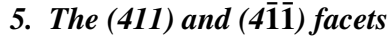

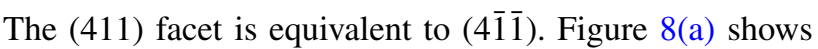
an STM image from the (411) facet. The step height (not shown) is $3.5 \pm 0.2 \AA$, which is close to the periodicity expected along [411], i.e., $a / \sqrt{18}$. As the regular features in STM are of about $10 \AA$ in diameter, no atomic resolution is obtained, and it is not therefore possible to associate the terrace in STM with an atomic plane of the model. However, the symmetry and size of the unit cell of the STM image can be compared with that of the (411) plane of the bcc lattice. In order to determine the unit cell, we analyze the autocorrelation function of the image [Fig. 8(b)], in addition to the FFT analysis discussed above. The autocorrelation was calculated using a function, $f(\mathbf{r})=\sum_{\mathbf{r}^{\prime}} f\left(\mathbf{r}^{\prime}\right) f\left(\mathbf{r}^{\prime}+\mathbf{r}\right)$, where $f\left(\mathbf{r}^{\prime}\right)$ is the image matrix. The maxima in the autocorrelation function reflect the periodicity of the image. The unit cell is found to be oblique with dimensions $(25.5 \pm 0.3 \AA, 21.5 \pm 0.1 \AA$, $\alpha=65^{\circ}$ ), which is consistent with that of the (411) plane of the Ag-In-RE $1 / 1$ approximant lattice, $\left(25.82 \AA, \alpha=50.5^{\circ}\right)$ [Fig. 8(c)].

\section{The (710) facet}

As with the (411) facets, the (710) facets do not show atomic resolution in STM. The smallest observed features
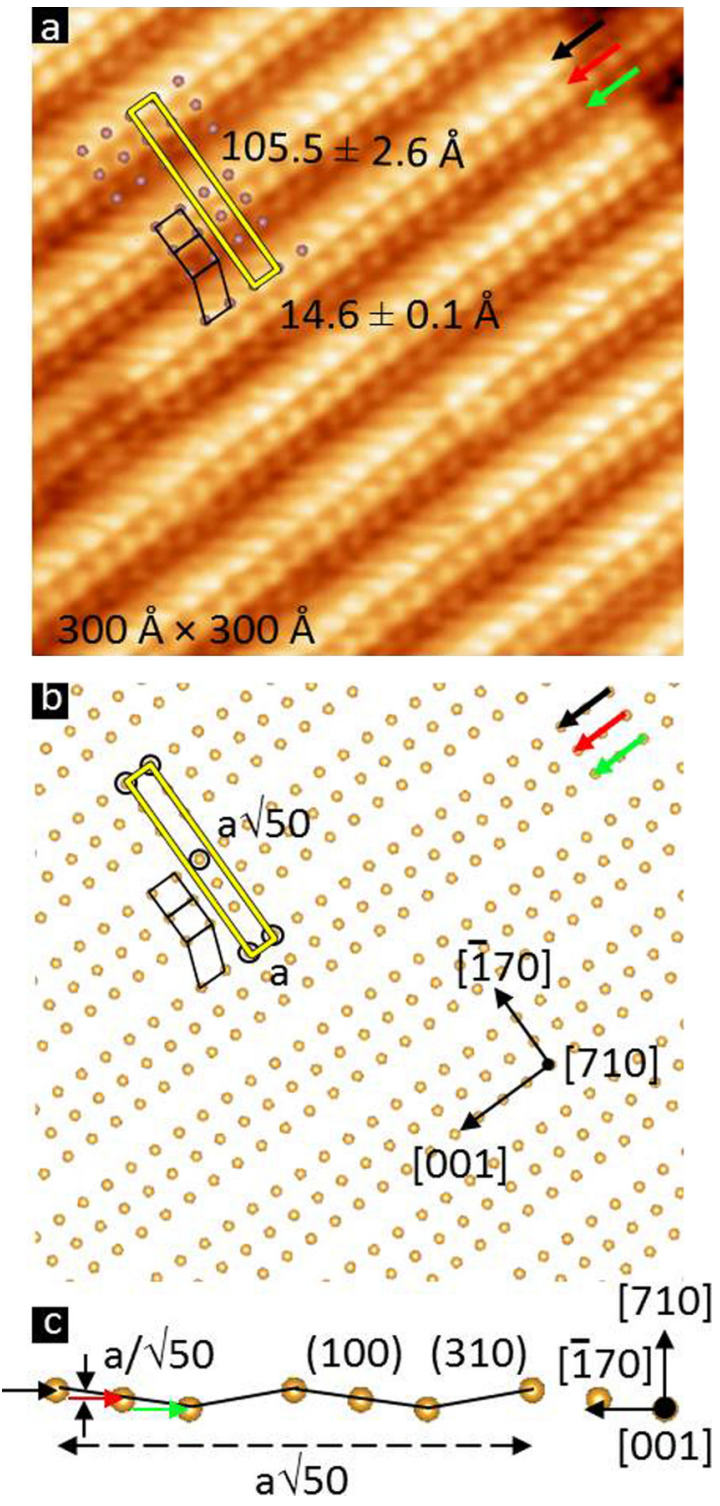

FIG. 9. (710) facet. (a) STM image from the (710) facet in the Ag-In-Tb 1/1 approximant $(300 \times 300 \AA)$. (b) The bcc space lattice cleaved and projected along [710]. (c) A portion of the bcc lattice (b) projected normal to [710].

are protrusions of $\approx 10 \AA$ size, which form parallel rows [Fig. 9(a)]. Rows of three different heights bunch together. These are marked with arrows with different colors, with the row indicated by the black arrow being measured at the top. Although lack of atomic resolution does not allow identification of the STM images with specific bulk atomic planes, the symmetry and size of the unit cell identified in STM are similar to that expected from the (710) plane of the bcc lattice, with a lattice constant of $15.57 \AA$. The (710) plane of the bcc lattice is shown in Fig. 9(b), which is a vicinal plane consisting of the narrow (100) and (310) planes [Fig. 9(c)]. The unit cell is a centered rectangle with dimension of $(a, \sqrt{50} a)$, which is in agreement with the unit cell observed in STM $(14.7 \pm 0.1 \times 105.5 \pm 2.6 \AA)$. The symmetry of the (001) and (310) planes is also identified in STM. 

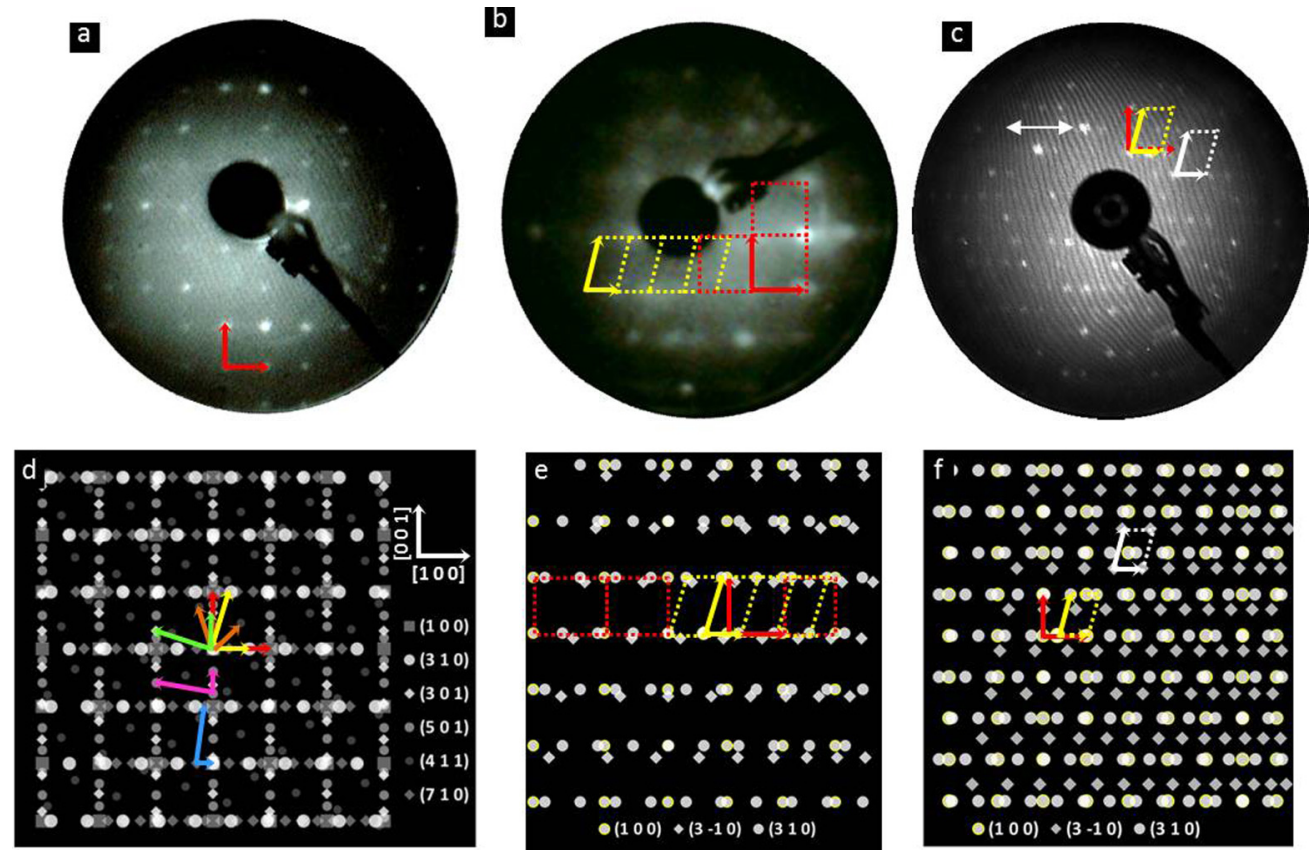

FIG. 10. LEED patterns from (a) (100) surface of the Ag-In-Yb 1/1 approximant (beam energy $32 \mathrm{eV}$ ) and (b), (c) the Ag-In-Tb 1/1 approximant [(b) $18 \mathrm{eV}$ and (c) $34 \mathrm{eV}$ ]. Patterns (b) and (c) were taken in different experimental chambers. (d) Reciprocal lattice of various facets of the ideal bcc lattice without considering angle of inclination of the facets. Reciprocal lattice vectors of each facet are shown by arrows. (e), (f) Simulated LEED patterns considering (310) is normal to the incident beam [(e) $18 \mathrm{eV}$ and (f) $34 \mathrm{eV}]$. Only reciprocal lattices of the facets relevant to the observed LEED patterns are shown.

\section{Low-energy electron diffraction (LEED)}

In order to crosscheck the formation of facets observed by STM, we employed LEED. Figure 10(a) shows a LEED pattern taken from the Ag-In-Yb sample, which was machine cut along (100) without a miscut and thus no surface miscut was introduced. As expected, all observed LEED spots belong to the (100) surface and no facets are observed. However, LEED patterns from the other two samples, such as those shown in Figs. 10(b) and 10(c), which were taken from AgIn-Tb, are more complicated.

LEED patterns from the faceted surfaces were heavily dependent on the tilt of the sample from the incident electron beam, the beam energy, and to some extent the position of the sample. We checked the patterns across the surface and tried to align the beam normal to one of the facets. However, our experimental setup allowed us to change the tilt in only one direction, so that an optimum alignment was not always achieved. However, by comparing with simulated Bragg patterns, the LEED patterns could be assigned to different facets.

Figures 10(d)-10(f) show schematics of the reciprocal lattices expected from various facets of the ideal bcc structure, without considering the inclination of the facets. However, the introduction of a tilt of the facets alters the reciprocal lattices significantly. We simulated LEED patterns for various tilt angles of the facets and orientations of the surface normal to the beam. The resulting patterns were carefully compared with the relative change in spot position in LEED as a function of energy. Our simulations could produce almost all patterns observed by LEED. Here we show two examples. The LEED pattern shown in Fig. 10(b) can be explained if the (310) facet is normal to the beam and only the (100) and (310) facets contribute to LEED. Similarly, strong spots of the LEED pattern in Fig. 10(c) can be assigned to the (310) and (310) facets with the (310) facet being normal to the beam.

Based on the miscut direction, the (501) facet should dominate because the macroscopic orientation of the surface is close to this direction [refer to Fig. 1(c)]. However, the (310) and (100) facets dominate in both STM and LEED measurements, suggesting that these facets are more energetically favored than others.

There were some features in the LEED patterns which could not be explained by bulk truncation. Particularly, very weak spots are observed between the rows of strong spots of (310) [their position is indicated by a double-headed arrow in Fig. 10(c)]. In the given image, these spots overlap with the $(3 \overline{1} 0)$ pattern, but are distinct at other beam energies (not shown here). These spots move coherently with the (310) spots upon change in beam energy. This can be explained if there is a $(2 \times 1)$ superlattice in the $(310)$ facet. The supercell could not be seen in STM images. Such a supercell could be produced by a chemical order of In and Ag in the neighboring RTH clusters or orientation order of the innermost tetragonal shell. The innermost tetrahedron is believed to be disordered or replaced by a single atom in some isostructural systems [7]. However, the chemical order could not be confirmed because of the lack of an atomic model of the Ag-In-RE approximants.

\section{DISCUSSION}

We now analyze various factors that may explain the stability of the observed facets. These include atomic density, 
TABLE II. Density and composition of the facet planes.

\begin{tabular}{|c|c|c|c|c|c|c|}
\hline \multirow[b]{2}{*}{ Facets } & \multirow{2}{*}{$\begin{array}{c}\text { Angle of inclination } \\
\text { with (100) }\end{array}$} & \multirow[b]{2}{*}{$d_{h k l} / a$} & \multicolumn{2}{|c|}{$\mathrm{Ag}-\mathrm{In}-\mathrm{Yb}$ density (atom/AA $\left.{ }^{2}\right)$} & \multicolumn{2}{|c|}{ Composition } \\
\hline & & & Topmost layer & With subsurface & Topmost layer & With subsurface \\
\hline (100) & $0^{\circ}$ & 0.5 & 0.0824 & 0.16085 & $\mathrm{Ag}_{43} \mathrm{In}_{37} \mathrm{RE}_{20}$ & $\mathrm{Ag}_{49} \mathrm{In}_{41} \mathrm{RE}_{10}$ \\
\hline (310) & $18.4^{\circ}$ & 0.3162 & 0.0208 & 0.01154 & $\mathrm{Ag}_{40} \mathrm{In}_{60} \mathrm{RE}_{0}$ & $\mathrm{Ag}_{44} \mathrm{In}_{34} \mathrm{RE}_{22}$ \\
\hline (301) & $18.4^{\circ}$ & 0.3162 & 0.0078 & 0.0466 & $\mathrm{Ag}_{33} \operatorname{In}_{67} \mathrm{RE}_{0}$ & $\mathrm{Ag}_{44} \mathrm{In}_{37} \mathrm{RE}_{19}$ \\
\hline (411) & $19.5^{\circ}$ & 0.2357 & & & & \\
\hline$(501)$ & $11.3^{\circ}$ & 0.1961 & 0.0122 & 0.07603 & $\mathrm{Ag}_{33} \mathrm{In}_{40} \mathrm{RE}_{27}$ & $\mathrm{Ag}_{43} \mathrm{In}_{36} \mathrm{RE}_{21}$ \\
\hline (611) & $13.3^{\circ}$ & 0.1622 & & & & \\
\hline (710) & $8.1^{\circ}$ & 0.1414 & & & & \\
\hline
\end{tabular}

chemistry, and bonding between atoms in the surface and subsurface regions.

We first calculate the atomic density of a simple bcc crystal, i.e., each lattice point associated with a single atom, using the fact that the density in a plane $(h k l)$ is proportional to the interplanar spacing $\left(d_{h k l}\right)$. For our analysis, we consider Miller planes with indices up to (999) and those of which make an angle up to $20^{\circ}$ with the (100) plane, i.e., an angle close to the maximum inclination of the observed facets. The planes are then sorted in a descending order of the density (Table II). We have omitted the equivalent planes in the table. We find that the planes with high density correspond to the observed facets, with the exception that the (611) facet is not seen in STM. The sum of the Miller indices of these high-density planes is an even number, except for (100). If the sum is odd, the density would be much lower. Thus, it can be inferred that the facets prefer to form at these high-density planes.

However, the bcc lattice of the current system is associated with an RTH cluster with a complex atomic distribution. Therefore, it is interesting to compare the atomic density in the approximant and a simple bcc crystal. It is not straightforward to define an atomic plane in a complex crystal, where atoms are discretely distributed in the crystal, unlike in a simple crystal where atomic planes are well separated, at least the low-index planes. We hypothesize that atoms within a certain thickness form a plane. We calculated the density of planes with different thickness of 0.3 and $0.7 \AA$. It was found that the calculated atomic density, in two cases, has a positive correlation with the density in a simple bcc crystal. However, the atomic density of the plane is much smaller than in a normal metal. For example, the density of the (111) surface of elemental fcc $\mathrm{Tb}$ is 0.178 atoms $/ \AA^{2}$. The density of the (100) plane in the Ag-In-Tb approximant is only half of this value. The (100) plane has the highest atomic density among the observed atomic planes. The density of the other observed facet planes is lower than (100) by a factor of four or more. As we will see below, atoms in the top surface layer are so far apart that they are less likely to be bonded together, i.e., they have to bond with subsurface atoms in order to be stabilized.

Before we discuss the bonding configuration of the surface atoms, we analyze the chemical composition of the surface layer, using information about the occupancy of $\mathrm{Ag}$ and In in different shells, as outlined in Sec. III A. The estimated composition of the topmost layer, i.e., the layer used to describe the STM images, is shown in Table II. As can be seen, the (100) surface has a composition similar to the bulk. The other facets are In rich in all facets. This is in agreement with observations on the Ag-In-Yb quasicrystal. All three high-symmetry surfaces (two-, three-, and fivefold) of this system are found to be terminated at a bulk plane containing In as the majority constituent [21-23]. We also present the density and composition of the surface region, including subsurface layers in Table III. All atoms in the subsurface which are likely to be bonded with the topmost surface layer are considered in the calculations (see discussion below about the bonding configuration). As can be seen, the atomic density in the surface region of (100) is comparable to that of fcc $\mathrm{Tb}$, but other facets still have lower density. The content of $\mathrm{Ag}$ increases in the subsurface region.

Finally, we discuss the bonding between surface atoms. For this, we determine the nearest-neighbor species and their separation in the top surface layer (Table II). For instance, the minimum separation of the nearest neighbors (NN) in the (310) facet (Fig. 5) is $2.98 \AA$, which is a distance between the $\mathrm{Ag}$ and $\mathrm{Ag}$ atoms. Both atoms belong to the midedge of the fifth shell. Therefore, these atoms could be identified

TABLE III. Distance between atoms in the topmost and subsurface layers of the Ag-In-Tb 1/1 approximant. The subsurface atoms are marked bold. The number in parentheses corresponds to occupancy of atoms. Refer to text for further discussion of occupancy.

\begin{tabular}{lcc}
\hline \hline Facets & Distance $(\AA)$ & $\operatorname{Atoms}$ \\
\hline$(100)$ & 2.81 & $\operatorname{Ag}(1)-\operatorname{Ag}(0.63) \operatorname{In}(0.37)$ \\
& 3.28 & $\operatorname{Tb}(1)-\mathbf{A g}(\mathbf{0 . 2}) \operatorname{In}(\mathbf{0 . 8})$ \\
$(310)$ & 3.33 & $\operatorname{Tb}(1)-\operatorname{Ag}(0.6) \operatorname{In}(0.4)$ \\
& 2.94 & $\operatorname{Ag}(0.2) \operatorname{In}(0.8)-\mathbf{A g}(\mathbf{0 . 6 3}) \operatorname{In}(\mathbf{0 . 3 7})$ \\
& 2.94 & $\operatorname{Ag}(0.2) \operatorname{In}(0.8)-\operatorname{Ag}(0.2) \operatorname{In}(0.8)$ \\
& 3.02 & $\operatorname{Ag}(0.2) \operatorname{In}(0.8)-\operatorname{Ag}(0.63) \operatorname{In}(0.37)$ \\
& 2.98 & $\operatorname{Ag}(1)-\operatorname{Ag}(1)$ \\
& 3.28 & $\operatorname{Ag}(0.2) \operatorname{In}(0.8)-\mathbf{T b}(\mathbf{1})$ \\
$(301)$ & 3.38 & $\operatorname{Ag}(0.2) \operatorname{In}(0.8)-\mathbf{T b}(\mathbf{1})$ \\
& 3.41 & $\operatorname{Ag}(1)-\mathbf{T b}(\mathbf{1})$ \\
& 3.33 & $\operatorname{Ag}(0.6) \operatorname{In}(0.4)-\mathbf{T b}(\mathbf{1})$ \\
& 2.94 & $\operatorname{Ag}(0.6) \operatorname{In}(0.4)-\mathbf{A g}(\mathbf{0 . 2}) \operatorname{In}(\mathbf{0 . 8})$ \\
& 2.97 & $\operatorname{Ag}(0.2) \operatorname{In}(0.8)-\mathbf{A g}(\mathbf{0 . 2}) \operatorname{In}(\mathbf{0 . 8})$ \\
& 3.68 & $\operatorname{Ag}(0.2) \operatorname{In}(0.8)-\operatorname{Ag}(0.2) \operatorname{In}(0.8)$ \\
& 2.88 & $\operatorname{Ag}(0.6) \operatorname{In}(0.4)-\operatorname{Ag}(0.6) \operatorname{In}(0.4)$ \\
& 2.94 & $\operatorname{Ag}(0.6) \operatorname{In}(0.4)-\mathbf{A g}(\mathbf{0 . 2}) \operatorname{In}(\mathbf{0 . 8})$ \\
& 3.23 & $\operatorname{Tb}(1)-\mathbf{A g}(\mathbf{0 . 2}) \operatorname{In}(\mathbf{0 . 8})$ \\
& 3.23 & $\operatorname{Ag}(0.2) \operatorname{In}(0.8)-\mathbf{T b}(\mathbf{1})$ \\
\hline \hline
\end{tabular}


as Ag, i.e., the occupancy of these sites by $\mathrm{Ag}$ is $100 \%$. We will represent this atom by $\operatorname{Ag}(1)$, with the number in parentheses corresponding to the occupancy of the atomic site. Similarly, the maximum separation of the nearest neighbors in this facet is $6.24 \AA$, which is a distance between atoms in fourth shells, i.e., between $\mathrm{Ag} / \mathrm{In}-\mathrm{Ag} / \mathrm{In}$ atoms. As the fourth shell contains $20 \% \mathrm{Ag}$ and $80 \% \mathrm{In}$, as described in Sec. III A, we represent this NN pair by $\operatorname{Ag}(0.2) \operatorname{In}(0.8)-A g(0.2) \operatorname{In}(0.8)$. As the separation of $\mathrm{Ag}(0.2) \operatorname{In}(0.8)-\mathrm{Ag}(0.2) \operatorname{In}(0.8)$ is far larger than the atomic diameter of $\mathrm{Ag}$ or In (atomic diameters of $\mathrm{In}, \mathrm{Ag}$, and $\mathrm{Tb}$ are $3.12,3.30$, and $4.5 \AA$, respectively), it is less likely that these atoms will be bonded. These atoms must therefore be bonded with subsurface atoms. Therefore, we determine the nearest neighbor of each surface atom in the subsurface, which is shown in Table III. The shortest center-to-center distance of atoms is $2.94 \AA$, which is between $\operatorname{Ag}(0.2) \operatorname{In}(0.8)-A g(0.63) \operatorname{In}(0.37)$. However, if we consider the atomic diameter of atoms, the gap between $\operatorname{Ag}(0.2) \operatorname{In}(0.8)$ $\operatorname{Ag}(0.63) \operatorname{In}(0.37)$ will be larger than $\operatorname{Ag}(0.2) \operatorname{In}(0.8)-\mathrm{Tb}(1)$ because $\mathrm{Tb}$ atom is larger than In by $40 \%$. Therefore, a bond is likely to occur between $\operatorname{Ag}(0.2) \operatorname{In}(0.8)-\mathrm{Tb}(1)$.

From a similar analysis of bonding in the other facets, we can extract common features. The atoms in the fourth shell, which are mostly In, are likely to be bonded with third shell Tb atoms, in the (100), (301), and (501) facets. In the (301) facet, the $\mathrm{Tb}$ atom is likely to be bonded with atoms in the second shell, which has $40 \%$ of In. Ag/In-Ag/In bonds also occur in all surfaces, but these bonds will be a minority. Therefore, we can conclude that the majority constituent of the surface, In, is bonded with $\mathrm{Tb}$.

The observation of the presence of the RE element in the surface is consistent with results of theoretical simulations. The STM simulation of the Ag-In-Ca 1/1 approximant showed that the $s p-d$ hybridization between the Cd-5p states and the low-lying $d$ states of $\mathrm{Yb} / \mathrm{Ca}$ plays an essential role in the stability of surface [20].

\section{CONCLUSIONS}

A surface study of the Ag-In-RE (RE: Gd, Tb, and $\mathrm{Yb}$ ) 1/1 approximants by STM and LEED has been presented. The surfaces of Ag-In-Gd and Ag-In-Tb produce facets after sputtering and annealing under ultrahigh-vacuum conditions, while the Ag-In-Yb approximant exhibits a flat (100) surface with no facets. We find that the faceting follows a miscut introduced during initial sample preparation and therefore likely has its origin therein. Both STM and LEED measurements confirm that the facets are formed along various

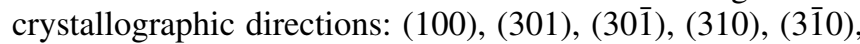
(411), (41) 1 ), (501), and (710). Most of the facets exhibit a step-terrace structure. The structure on the terraces can be related to a truncation of the rhombic triacontahedral clusters, which are the building blocks of these systems.

We analyze various factors that may influence the selection of the facet planes. These factors include atomic density, chemistry, bonding between atoms, and symmetry of the facet planes. We find that despite having different crystallographic orientation, the facets exhibit common features.

The facet planes are found to be rich in In. No surface segregation occurs, although the surfaces selected are In rich. The analysis based on nearest-neighbor distance and atomic radii indicates that In atoms are likely to be bonded with $\mathrm{RE}$ atoms. The atomic density of the topmost layer of the facet planes is much less than that of close-packed fcc (111) Tb, such that the nearest-neighbor distance of atoms is much larger than the atomic diameter. Therefore, these atoms have to be bonded with the subsurface atoms, indicating the role of subsurface atoms in the surface stability. On the basis of our observation of $\mathrm{Yb}$-In bonding and the previous report on STM simulation on the Ag-In-M (M: metal) 1/1 approximation, we suggest that the hybridization between electronic states of $\mathrm{Ag} / \mathrm{In}$ and RE atoms plays a role in the stability of the surface. Our work demonstrates the inherent complexity in characterization of the surface structure of the complex intermetallic alloys.

\section{ACKNOWLEDGMENTS}

Support from the Engineering and Physical Sciences Research Council (Grant No. EP/D071828/1) is gratefully acknowledged. The authors thank M. Shimoda for providing a program to analyze the atomic structure and density of the Ag-In-RE 1/1 approximant. Part of this work is a result of the cooperation within the European C-MAC. T.P.Y. acknowledges the financial support from DST, Government of India for the BOYSCAST fellowship, and Banaras Hindu University for study leave. S.S.H. is grateful to the Kurdistan Regional Government, Ministry of Higher Education and Scientific Research, University of Raparin for financial support for his Ph.D.
[1] J. Dshemuchadse, D. Y. Jung, and W. Steurer, Acta Crystallogr. Sec. B 67, 269 (2011).

[2] D. Shechtman, I. Blech, D. Gratias, and J. W. Cahn, Phys. Rev. Lett. 53, 1951 (1984).

[3] A. P. Tsai, J. Guo, E. Abe, H. Takakura, and T. J. Sato, Nature (London) 408, 537 (2000).

[4] J. Q. Guo, E. Abe, and A. P. Tsai, Phy. Rev. B 62, R14605 (2000).

[5] C. Cui and A. P. Tsai, Philos. Mag. 91, 2443 (2011).

[6] R. Tamura (private communication).

[7] G. Gebresenbut, M. S. Andersson, P. Beran, P. Manuel, P. Nordblad, M. Sahlberg, and C. P. Gómez, J. Phys. Condens. Matter 26, 322202 (2014).
[8] T. Hiroto, K. Tokiwa, and R. Tamura, J. Phys. Condens. Matter 26, 216004 (2014).

[9] H. Takakura, C. P. Gómez, A. Yamamoto, M. de Boissieu, and A. P. Tsai, Nat. Mater. 6, 58 (2006).

[10] M. de Boissieu, H. Takakura, C. P. Gòmez, A. Yamamoto, and A. P. Tsai, Philos. Mag. 87, 2613 (2007).

[11] M. Armbrüster, K. Kovnir, M. Friedrich, D. Teschner, G. Wowsnick, M. Hahne, P. Gille, L. Szentmiklosi, M. Feuerbacher, M. Heggen, F. Girgsdies, D. Rosenthal, R. Schlogl, and Y. Grin, Nat. Mater. 11, 690 (2012).

[12] H. R. Sharma, K. Nozawa, J. A. Smerdon, P. J. Nugent, I. McLeod, V. R. Dhanak, M. Shimoda, Y. Ishii, A. P. Tsai, and R. McGrath, Nat. Commun. 4, 2715 (2013). 
[13] J. A. Smerdon, K. M. Young, M. Lowe, S. S. Hars, T. P. Yadav, D. Hesp, V. R. Dhanak, A. P. Tsai, H. R. Sharma, and R. McGrath, Nano Lett. 114, 1184 (2014).

[14] V. Fournée, E. Gaudry, J. Ledieu, M.-C. Weerd, D. Wu, and T. Lograsso, ACS Nano 8, 3646 (2014).

[15] C. Cui and A. P. Tsai, J. Alloys Compd. 536, 91 (2012).

[16] I. Horcas, R. Fernandez, J. M. Gomez-Rodriguez, J. Colchero, J. Gomez-Herrero, and A. M. Baro, Rev. Sci. Instrum. 78, 013705 (2007).

[17] K. Momma and F. Izumi, J. Appl. Crystallogr. 44, 1272 (2011).

[18] C. P. Gòmez (private communication).
[19] C. Cui, H. R. Sharma, P. J. Nugent, M. Shimoda, and A. P. Tsai, Acta Phys. Polon. A 126, 577 (2014).

[20] K. Nozawa and Y. Ishii, J. Phys.: Conf. Ser. 226, 012030 (2010).

[21] H. R. Sharma, M. Shimoda, K. Sagisaka, H. Takakura, J. A. Smerdon, P. J. Nugent, R. McGrath, D. Fujita, S. Ohhashi, and A. P. Tsai, Phys. Rev. B 80, 121401 (2009).

[22] C. Cui, P. J. Nugent, M. Shimoda, J. Ledieu, V. Fournée, A. P. Tsai, R. McGrath, and H. R. Sharma, J. Phys. Condens. Matter 24, 445011 (2012).

[23] C. Cui, P. J. Nugent, M. Shimoda, J. Ledieu, V. Fournée, A. P. Tsai, R. McGrath, and H. R. Sharma, J. Phys. Condens. Matter 26, 015001 (2014). 